\title{
Tomadas de decisão baseado em análises estratégicas: estudo de caso em uma microempresa de biotecnologia
}

\author{
Paulo Henrique de Lima Siqueira* \\ Felipe Alexandre de Souza Félix Nunes**
}

\begin{abstract}
RESUMO
A estratégia é um conceito essencial em qualquer empresa, de qualquer porte. No entanto é preciso entender melhor o impacto do uso das ferramentas de análise para formulação de um planejamento para Micro e Pequenas Empresas. O objetivo desse traballho foi o de compreender os processos de administração estratégica como meios de alavancar o desempenho de micro e pequenas empresas, observando a utilização de ferramentas de análise das estratégias por uma Micro Empresa do setor de biotecnologia, que tem como base o uso de novas tecnologias para criação de vantagem competitiva. Os resultados desse estudo de caso mostraram que, após identificar as oportunidades, a empresa estudada fez um contrato de prestação de serviços técnicos com a financiadora do novo projeto, que precisou recorrer aos conhecimentos oferecidos para introduzir um novo sistema de diagnóstico no mercado. A proposta em questão oferecia a firma estudada acesso à tecnologia exclusiva, em um segmento com altas barreiras de entrada, em contrapartida a empresa utilizaria o alto grau de conhecimento técnico aliado à compreensão do funcionamento da complexa legislação brasileira para lançamento de produtos no setor de saúde pública.
\end{abstract}

Palavras-chave: análise estratégica; estratégia; micro e pequena empresa.

\section{DECISION BASED ON STRATEGIC ANALYSIS: A CASE STUDY IN A BIOTECHNO- LOGY MICROENTERPRISE}

\begin{abstract}
Strategy is an essential to small, medium and bigger companies. However, it is necessary to understand better the impact of using analysis tools to formulate planning for Micro and Small Enterprises. The objective of this work was to understand the strategic management processes as a means of leveraging the performance of micro and small companies, observing the use of strategy analysis tools by a Micro Company in the biotechnology sector, which is based on the use of new technologies to create competitive advantage. The results of this case study showed that, after identifying the opportunities, the studied company entered into a contract for the provision of technical services with the financier of the new project, which needed to use the knowledge offered to introduce a new diagnostic system in the market. The proposal in question offered the studied company access to exclusive technology, in a segment with high
\end{abstract}

\footnotetext{
* https://orcid.org/0000-0001-8204-7846. Universidade Federal de São João del Rei. Profesor Adjunto no departamento de ciências administrativas e contábeis da Universidade Federal de São João del Rei. E-mail: paulosiqueira@ufsj.edu.br

** CEPEAD/FACE - Universidade Federal de Minas Gerais - UFMG. Graduado em Engenharia de Produção pela Universidade Federal de São João del Rei e Mestrando. E-mail: fasfn@ufmg.br .
} 
entry barriers, in return the company would use the high degree of technical knowledge coupled with the understanding of the functioning of the complex Brazilian legislation to launch products in the health sector public.

Key-words: strategic analysis; strategy; micro and small business

\section{INTRODUÇÃo}

A história da industrialização do Brasil e as diversas políticas governamentais e econômicas configuraram uma estrutura peculiar de micro e pequenas empresas (MPE). Estas empresas são responsáveis pela ocupação de até $72 \%$ dos postos de trabalho formais gerados no país (SEBRAE, 2018). Em princípio o motivador da visão estratégica destas organizações é embasado na maneira de julgar e nas práticas dos precursores, usualmente um indivíduo, grupo familiar ou pequenas sociedades, e habitualmente dota de pouca ajuda de outros membros na condução, o que contribui para que a intuição e interesses particulares determinem a tomada de decisão na formulação da estratégia (GONÇALVES; KOPROWSKI, 1995).

O caminho do desenvolvimento operacional e produtivo é o desenvolvimento e multiplicação de novas tecnologias, normalmente aceito como fator de fomento às capacidades da organização, o termo inovação foi popularizado por Joseph Schumpeter que o usou para descrever novos elementos que movimentam o sistema capitalista (PAIVA et al., 2018). As inovações provocam impactos macroeconômicos variados, se tratando de empresa originam vantagens competitivas, para o nível econômico desenvolvem novos setores e na perspectiva da concorrência provocam sensíveis alterações na estrutura de mercado (FLEURY et al., 2016).

Não há relação clara entre o investimento em Pesquisa e Desenvolvimento (P\&D) e rentabilidade, ainda que o investimento em pesquisa e desenvolvimento fosse associado à melhoria subsequente (HARRIS; LENOX, 2013). Os autores reforçam a ideia afirmando a dificuldade em prever o Valor Presente Líquido (VPL) no futuro, a priori ao decidir investir em $P \& D$, muito porque os produtos inovadores muitas vezes não são obrigatórios e surgem sem aviso prévio.

Além da relação entre a formulação da estratégia e a capacidade de inovação, é preciso ambientar o cenário competitivo da indústria biotecnológica. A biotecnologia engloba esferas da economia, como saúde humana e animal, a produção vegetal, entre outros exemplos. O setor encontra-se entre as áreas que mais se utilizam de processos inovadores, permitindo o aperfeiçoamento de novos produtos e procedimentos no ramo diagnóstico até a manipulação de animais e plantas geneticamente modificados. A capacidade de inovação é o elemento característico da competência para a firma manter-se neste cenário (MOLINA PALMA, 2004).

Para se adaptar a este ambiente de competitividade, Cavalcanti et al. (2016), sugerem a necessidade de melhorar a capacidade de planejamento, considerando o mercado e o cliente, utilizando a abordagem analítica. Desta forma, num ambiente inovador, onde os recursos não são abundantes, a análise da estratégia assumida define quais devem ser as correções no planejamento, o que pode ser implantado como melhoria e o que deve ser descartado para a contenção de gastos. 
Frente ao apresentado, este trabalho pretende compreender os processos de administração estratégica como meios de alavancar o desempenho de micro e pequenas empresas. Desta maneira se observam a utilização de ferramentas de análise das estratégias utilizadas por uma Micro Empresa do setor de biotecnologia, que tem como base o uso de novas tecnologias para criação de vantagem competitiva.

Para tal será explorado o conceito de estratégia e sua utilização neste mercado, os fatores de inovação que se aliam no diferencial mercadológico, e avaliar por meio de técnicas as estratégias utilizadas nos últimos anos.

Foram realizadas observações in loco e entrevistas semiestruturadas com os gestores da microempresa estudada. Outros dados como relatórios internos e planilhas financeiras foram disponibilizadas, por este motivo não é permitido citar a empresa ou revelar informações detalhadas sobre concorrentes, fornecedores e outros agentes participantes da observação.

\section{REFERENCIAL TEÓRICO}

\subsection{Inovação}

Uma explicação do termo inovação refere-se ao Manual de Oslo, declaração publicada pela OCDE (Organização para a Cooperação e Desenvolvimento Econômico) (2006), que define o termo como a implantação de uma novidade organizacional, seja ela uma prática de negócio, produto ou ação mercadológica, que se traduza em benefício para a instituição.

O impulso fundamental que põe e mantém em funcionamento a máquina capi-
talista procede dos novos bens de consumo, dos novos métodos de produção ou
transporte, dos novos mercados e das novas formas de organização industrial
criadas pela empresa capitalista (...) Este processo de destruição criadora é bá-
sico para se entender o capitalismo. É dele que se constitui o capitalismo e a ele
deve se adaptar toda a empresa capitalista para sobreviver. (SCHUMPETER,
1961, p. 110, grifo nosso).

Assim, a inovação tem se tornado cada vez mais importante para a estratégia das empresas. De acordo com Tessarin et al. (2019), apesar da taxa de inovação ser modesta no Brasil (36\%), essas empresas detêm cerca de 67\% das receitas líquida de vendas. Para as pequenas empresas, inovação é um requisito fundamental para alcance de viabilidade a longo prazo, influenciando fortemente seu sucesso ou fracasso (DUNNE et al., 2016).

Alguns autores atrelam a inovação de produto a uma oportunidade de mercado. Stefanovitz et al. (2014) acreditam que, nesta concepção é fundamental considerar a importância de a organização estar conectada ao meio externo para ser inovadora, pela necessidade de adquirir conhecimento e avaliar tendências mercadológicas, por tudo isso é preciso conceber alianças com as mais diversas fontes de conhecimento em determinados setores.

Em geral, há a consciência de que a inovação deve ser utilizada como vantagem competitiva estratégica. Desta forma, precisam-se avaliar os tipos de transformação implantados, quais os riscos e assim desenhar o melhor estratagema. É importante que se entenda a mudança como processo, e preservar o fluxo contínuo de evolução nos diversos 
setores da firma. Uma liderança habilidosa tem de garantir o ambiente propício, esse clima organizacional correto requer autonomia, incentivos e fluxo livre de informações para conciliar as divergências e ser capaz de chegar a essas ideias criativas que vão conduzir o processo de inovação.

\subsection{Estratégia}

Revisões bibliográficas recentes estruturaram a influência das publicações sobre pesquisas em estratégia (FERREIRA; FERNANDES; RATTEN, 2016). Dois textos são as bases das discussões em estratégia, o trabalho de Barney (1991) uma referência da visão baseada em recursos (VBR) e o livro de Michael Porter (1980) como um dos mais influentes estudiosos da área, lançando a sua visão baseada na indústria, fundamentada na Economia da Organização Industrial (EOI).

Para Porter (1997) numa empresa diversificada há duas categorias de estratégia: (i) a estratégia em nível corporativo, ou seja, a estratégia da empresa como um todo; e (ii) a estratégia de unidade de negócios, também chamada estratégia competitiva. Já Mintzberg e Quinn (1991) incluem também o nível de estratégia funcional, que determina como uma área operacional deve trabalhar a partir da estratégia de negócio escolhida.

No âmbito das estratégias funcionais de acordo com Lobato et al. (2003), a forma de atuação de uma área funcional da firma, sendo relacionado ao nível tático da organização. Neste nível de atuação, definitivamente operacional, o uso de algumas ferramentas torna-se importante para controle das ações tomadas, desta forma é essencial à firma prezar pela utilização de indicadores de recursos e desempenho que apontam os resultados das operações e indicam quais correções precisam ser realizadas.

Neste conceito o mapeamento de processos torna-se fundamental para compreender os procedimentos e o fluxo de informação e recursos por meio dos processos operacionais e da cadeia interna de valores (CARPINETTI, 2000). Uma das ferramentas mais utilizadas na modelagem de processos e recursos tem sido o Enterprise Resource Planning (ERP), que na elucidação de Souza e Zwicker (2000) são sistemas de informação integrados, obtidos como softwares, objetivando sustentar a maior parte das operações da firma..

\subsection{Análise estratégica}

Para que uma ideia seja bem-sucedida é necessário entender a concorrência e a dinâmica competitiva ao longo do tempo, e fazê-lo com as complexidades e incertezas do mundo real, para em seguida fundamentar a análise em dados gerados e raciocínio lógico. Por fim, a avaliação, propriamente dita, se baseia nos estudos do ambiente competitivo, no posicionamento da firma frente a esta competição e no diagnóstico das decisões estratégicas, utilizando ferramentas para avaliar o impacto de cada uma delas nas diretivas da organização.

A primeira atividade do processo de planejamento estratégico, conforme afirma Harris e Lenox (2013), é a análise ambiental. Nesta conjuntura, são apuradas as competências (pontos fortes) e as fragilidades (pontos fracos) pertinentes à organização, que representa- 
rão as suas capacidades de competir. É importante então que, uma vez identificada essas capacidades cruciais para a organização, dê-se processamento na intenção de desenvolver a fonte de tais capacidades.

Harris e Lenox (2013) debatem sobre a distinção entre capacidades e recursos, classificando o primeiro termo como o potencial que pode ser alcançado pela firma, e o segundo termo como os bens que propiciam este avanço, classificados como tangíveis (como colaboradores talentosos e patentes) ou intangíveis (marca, reputação e lealdade dos clientes). Desta forma, a primeira etapa ao analisar as capacidades da firma é usar a cadeia de valores para articular as habilidades da organização e, então, destrinchar estas habilidades, por meio da compreensão das pessoas, processos e sistemas, que dão início a competência para entregar estas capacidades.

Rocha e Borinelli (2007) afirmam que a Cadeia de Valor é uma sequência de atividades que se inicia com a origem dos recursos indo até o descarte do produto pelo último consumidor, separando as atividades de destaque estratégico de forma a compreender o papel dos custos e as fontes existentes e potenciais de diferenciação. Apresentado o aspecto teórico da Cadeia de Valor é preciso conceituar e estabelecer o papel dos stakeholders na configuração da organização, e sua participação na tomada de decisão estratégica. São indivíduos, grupos ou organizações, envolvidos por comportamentos praticados para geração de valor para fornecedores, clientes, empregados, governo e acionistas de forma que as empresas criadoras de valor baseiam suas decisões em manter uma proposição equilibrada entre os interesses competitivos de todos esses agentes (IMA, 1997).

A análise de stakeholders é qualificada como dinâmica, sendo que não considera as variáveis ambientais como passivas uma vez que entende que muitas delas são oriundas das ações e reações destes agentes. É dever das instituições preservar um relacionamento conveniente com os diferentes grupos de stakeholders, de acordo com o nível de importância na Cadeia de Valor, sendo: (i) stakeholders primários, os considerados mais influentes; e (ii) stakeholders secundários, os que influenciam menos na geração de valor (WOOD, 1990).

No seguimento das análises realizadas na empresa estudada, aprofunda-se na avaliação de um dos agentes da Cadeia de Valor, conceituando o estudo dos competidores. Na visão de Aaker (2009) a importância de entender os concorrentes faz-se justificada em quatro conceitos: (i) o conhecimento dos pontos fortes e francos da estratégia atual de um competidor torna possível captar oportunidades e ameaças que requerem uma resposta; (ii) deter notícias sobre futuras estratégias dos adversários pode prever ameaças e oportunidades emergentes; (iii) tomar decisões sobre estratégias opcionais consegue complementar a eficiência de prever a resposta provável dos competidores relevantes; (iv) a análise dos competidores pode ocasionar o reconhecimento de algumas incertezas estratégicas, que devem ser vigiadas incisivamente durante algum tempo.

A utilidade de conseguir informações do ambiente externo à firma e absorver ao processo de tomada de decisão é alusiva ao crescimento de uma área intitulada Inteligência Competitiva (IC). Canongia et al. (2004), afirmam que a IC é uma ferramenta habitualmente utilizada pelas organizações para eticamente detectar, recolher, estruturar e analisar 
informações relevantes sobre seu ambiente concorrencial, garantindo maior segurança à tomada de decisão da instituição.

Por fim, na contextualização das ferramentas analíticas tem-se a análise de cenários, que segundo Fahey e Randall (1998) são descrições de futuros possíveis, baseados em decisões assumidas na atualidade, não sendo consideradas adivinhações ou estratégias, contudo são hipóteses de crescimento, produzidas para perceber determinados riscos e oportunidades envolvidas nas estratégias de desenvolvimento.

Com o surgimento de novas tecnologias esta perspectiva foi sendo alterada com o pensamento que, ao invés de determinar o futuro de forma precisa para tomar decisão, era mais importante dotar as decisões presentes de conhecimento sobre as possibilidades do futuro. Essa diferença determinou o conceito sobre prospecção tecnológica e o modelo de trabalho de suas ferramentas na gestão da inovação, determinando a nova abordagem com o nome de Technology Foresight, ou simplesmente Foresight. (CANONGIA et al., 2004).

Os autores Santos e Santos (2003) definem o termo Foresight como uma atividade que harmoniza três aspectos distintos em um único processo: (i) pensar o futuro, examinando possíveis eventos a partir da propensão de longo prazo e argumentos sobre fatos novos e abruptos; (ii) discutir o futuro, requerendo a participação de diversos stakeholders e suas percepções sobre as tendências vindouras; e (iii) forjar o futuro, baseando-se na detecção de futuros possíveis e desejáveis, levando em conta a comunicação entre os agentes da Cadeia de Valor, que produzem, progressivamente, decisões em diferentes níveis.

\section{Metodologia}

O objetivo deste estudo foi buscar a compreensão da utilização de ferramentas de análise e dos processos de concepção e estruturação da estratégia de uma MPE, optando-se, neste caso, por uma pesquisa empírica do tipo estudo de caso. Essa opção se deu para compreender o fenômeno de maneira empírica, dentro do contexto da vida real, de forma a prover riqueza de dados e permitir a investigação de desafios gerenciais contemporâneos (YIN, 2015).

De acordo com Voss et al. (2002), esta aplicação é uma poderosa metodologia de pesquisa nos casos de gestão operacional. Seu uso é indicado em quatro diferentes ocasiões: (i) exploração de áreas ainda não suficientemente trabalhadas; (ii) elaboração de uma nova teoria; (iii) expansão de um novo tratado; (iv) teste de uma teoria existente, sendo esta última utilização que se tratará este trabalho, partindo de uma teoria em voga e os resultados de sua aplicação em um ambiente industrial.

As informações necessárias para a formulação deste estudo foram obtidas por meio de entrevistas semiestruturadas com os responsáveis pelo gerenciamento estratégico da empresa. Foram entrevistados o diretor industrial e o diretor presidente, como característica de uma MPE, ambos centralizam o controle da tomada de decisão. As entrevistas foram gravadas e transcritas manualmente para posterior análise. Também como fontes de dados foram utilizados documentos da organização, a observação in loco, e informações de acesso público disponíveis no sítio da firma na Internet. 


\section{A EMPRESA}

$\mathrm{O}$ estudo de caso foi realizado em uma microempresa brasileira de biotecnologia focada no desenvolvimento e produção de soluções para automação laboratorial, mais especificamente, automação para análises clínicas. Por razão de confidencialidade denominada neste trabalho por EMPRESA. A missão da organização estudada é fornecer soluções customizáveis e com elevado conteúdo tecnológico para otimização em análises clínicas. A visão é ser referência no fornecimento de equipamentos e soluções inovadoras para automação em análises clínicas.

\subsection{A primeira formulação estratégica}

No início de 2012, a EMPRESA iniciou um trabalho de identificação das capacidades internas, das estruturas da indústria e a determinação do seu posicionamento competitivo, mapeando suas vantagens e desvantagens e esboçando a estratégia seguinte numa detecção das oportunidades e ameaças do mercado brasileiro de exames clínicos. A firma realizou a análise tendo como objetivo definir as características da organização para traçar o plano estratégico dos anos subsequentes.

Detalhadas as capacidades constatou-se que seu ativo mais importante era a perícia técnica do departamento de Pesquisa e Desenvolvimento, e que era preciso dar tempo para que os resultados dos investimentos naquele setor se transformassem em retorno financeiro. Ao mesmo tempo em que a maior fraqueza identificada era a estrutura limitada, que restringia as ações dos departamentos de produção, comercial e logística. Com isso a EMPRESA não tinha possibilidade de aumentar a quantidade de equipamentos produzidos, sua capacidade de distribuição e o alcance do mercado restrito, o que restringiu o crescimento da firma.

Partindo destas análises, já no final daquele ano, esta firma iniciou prospecções de empresas parceiras no exterior após identificar a demanda do mercado nacional, que apontava a oportunidade de crescimento do nicho de análise sanguínea por gasometria. A busca encontrou uma multinacional que pretendia introduzir os produtos no Brasil.

Por meio de um contrato de licenciamento com prazo definido de dois anos, a EMPRESA iniciou a produção e distribuição de equipamentos e insumos. Em contrapartida houve investimento para reestruturação da planta industrial, que aumentou a capacidade da produção e o desempenho do setor de logística. O objetivo desta aliança era dar entrada dos produtos da multinacional no país, que depois de estabelecida teria como opção assumir a planta e a produção dos equipamentos, comprando a participação brasileira do acordo.

A resposta imediata do mercado provocou um crescimento de $602,75 \%$ entre janeiro de 2013 e dezembro de 2014. Embora houvesse resultados expressivos a EMPRESA sofreu com problemas em atender a grande demanda gerada pela falta de controle e de previsão ideais. Dado a falta de alinhamento estratégico a multinacional mudou os termos e retirou-se da parceria o que comprometeu o objetivo final da EMPRESA. Essa modificação provocou queda dos resultados financeiros entre os anos de 2015 e 2016, seguintes ao final do acordo. 


\subsection{A formulação de uma nova estratégia}

A firma realizou uma avaliação da possibilidade de apostar uma segunda vez na estratégia de licenciamento para lançar um novo produto no mercado, visando alcançar outros nichos de testes médicos. Mapeou-se os competidores e aliado a uma análise de stakeholders, traçou-se os objetivos de uma nova aliança, definindo com exatidão o que precisava estabelecer para atender a expectativa do novo parceiro.

A firma detectou, em uma atualização de suas primeiras análises, que havia corrigido setores anteriormente deficitários. Se antes havia defasagem na Produção e na Logística, a implantação de um sistema de Enterprise Resource Planning (ERP) garantiu maior controle dos processos da firma. Ademais, a estrutura física da planta industrial foi modificada e setores como controle de qualidade e assessoria científica também foram desenvolvidos para assegurar padrões de segurança da ANVISA.

$\mathrm{Na}$ identificação das fraquezas a firma voltou a identificar uma falha nos processos do departamento comercial, o que comprometeu na avaliação do mercado, nas políticas de venda e no retorno financeiro. Houve a necessidade de estruturar o departamento, vital para as pretensões da companhia. Outras falhas mapeadas foram a instabilidade financeira gerada pelo turbulento movimento anterior, aliada à crise econômica que abateu sobre o país, uma assistência técnica insuficiente para a demanda e a sobrecarga de trabalho sobre o P\&D.

Dentre as avaliações do ambiente detectou-se oportunidade para a atração de investidores para projetos tecnológicos apoiando-se no know-how das normas de saúde e da capacidade técnica do $\mathrm{P} \& \mathrm{D}$. De encontro, as ameaças reveladas foram os rivais para o segmento do mercado, capazes de usarem as capacidades financeiras para barrar a entrada de um novo produto. Além disso, a crise econômica que afeta na captação de recurso e nas vendas para o consumidor final provocou outra das ameaças identificadas, a saída de funcionários capitais por falta de receita.

Percebeu-se novas capacidades notadas entre os recursos intangíveis como resultados do primeiro plano estratégico. O crescimento da marca no mercado de diagnósticos clínicos rendeu matéria de destaque em uma das revistas de negócios mais importantes do Brasil. E a experiência, com os acertos e erros da estratégia anteriormente traçada, preparou a empresa para as possíveis oscilações que pudessem defrontar.

Para a definição do movimento da EMPRESA realizou-se análise de competidores - identificando quatro players como gigantes do mercado e um novo player utilizando-se de estratégia semelhante à EMPRESA. Também se executou análise de cenários para um horizonte de evento de quatro anos, o que determinou enfoque em pequenos e médios laboratórios com estratégia de preços mais baixos que os concorrentes. Esse diagnóstico também baseou investida em licitações.

A análise de stakeholders considerou como atores fundamentais a empresa parceira, os sócios, os colaboradores e distribuidores. Outros elementos identificados como motivo de interesse foram hospitais, laboratórios e os pacientes. A firma acreditou que gerando valores para os stakeholders chave, por consequência geraria valores para estes atores secundários. 
A EMPRESA definiu como estratégia de crescimento o licenciamento em território brasileiro da produção e distribuição do equipamento e testes de marcadores cardíacos, produto este originalmente concebido por uma grande empresa estrangeira. $\mathrm{O}$ cenário envolveu poucos investimentos em infraestrutura, ao passo que um grande aporte na compra de insumos por meio de importações, para manufaturá-los em território nacional.

Configurado para enfrentar apenas um adversário direto no nicho de mercado que pretendia atender, a firma estudada definiu a relação com os distribuidores como primordial, visando criar uma vantagem competitiva oriunda desse envolvimento mais próximo. Assim como a utilização de pregoeiros, para encontrar editais e licitações.

A partir da liberação da vigilância sanitária do país, em maio de 2016, a venda dos produtos lançados pela EMPRESA teve índice de crescimento de 39,81\%. Infelizmente para a firma estudada o tempo de implantação da estratégia de licenciamento não era compatível com os recursos financeiros disponíveis, e o chamado "vale de receitas" indicava um ponto final nas pretensões e o fechamento do negócio.

\subsection{Negócio de oportunidade: crescimento pela inovação}

Por meio de uma oportunidade identificada nas análises estratégicas, motivada pelas capacidades da empresa, a EMPRESA identificou uma investidora, que precisou recorrer aos conhecimentos oferecidos para introduzir um novo sistema de diagnóstico no mercado. Desta forma foi estabelecido um contrato de prestação de serviços técnicos com a financiadora do novo projeto.

A proposta em questão oferecia a firma estudada acesso à tecnologia exclusiva, em um segmento com altas barreiras de entrada, em contrapartida a empresa utilizaria o alto grau de conhecimento técnico aliado à compreensão do funcionamento da complexa legislação brasileira para lançamento de produtos no setor de saúde pública.

A investidora parceira havia desenvolvido uma nova tecnologia para detecção de um agente infeccioso a partir de um teste rápido, entretanto, esbarrou na falta de conhecimento do mercado e das barreiras de entrada que abrange principalmente a legislação vigente. Desta maneira, a firma estudada entrou no negócio com a bagagem de ser uma empresa estabelecida no mercado, de já ter as licenças para produção de equipamentos médicos e com know-how para desenvolver a tecnologia necessária para a leitura dos testes.

Assim, aliando um projeto de desenvolvimento de um equipamento moderno, cuja funcionalidade complementa a tecnologia originalmente concebida pela parceira, a firma estudada criou condições para sobrevivência e estabelecimento dos novos produtos no mercado.

\section{CONSIDERAÇÕES FINAIS}

Este trabalho analisou a influência dos processos de administração estratégica como precursores do desempenho das micro e pequenas empresas. Teve como objetivo observar a necessidade da avaliação de fatores internos e externos para a formulação da estratégia de negócio. O resultado desse estudo salienta a valência do pensamento estratégico no intuito de equilibrar os parâmetros com as grandes forças do mercado. 
Tal investigação determina a pertinência de se elaborarem estratégias em micro e pequenas empresas de modo inteirado e sistêmico, abarcando eficiência de execução de todas as etapas do procedimento (análise, planejamento, implementação, controle e feedback). Isso reflete sobre o processo da análise e formulação da estratégia indicando que as informações produzidas nestas etapas se tornam o elemento primordial dos gestores na implantação do pensamento estratégico integrado.

Levando em consideração ausências existentes na literatura, que certifiquem empiricamente a relação entre gestão estratégica e desempenho de micro e pequenas empresas, e da relevância do assunto, nota-se, ainda que em caráter exploratório, a realização de um trabalho que verifique empiricamente esta correlação pode ser de grande valia, não só para gestores, mas também para acadêmicos, gerando estudos posteriores, que comprovem a hipótese em outros setores e também constatar empiricamente a possibilidade de generalizar seus resultados.

Para as firmas e gestores, este trabalho reforça a literatura e gera a sugestão de que pratiquem a administração estratégica em todas as suas fases. Por circunstâncias, defende-se que as micro e pequenas empresas não projetem ou ainda não atuem em uma gestão sistemática. Entretanto, ao observar a prática do pensamento estratégico por esta organização induz sobre a relevância da especialização e profissionalização da gestão, levando-se em consideração a importância socioeconômica das organizações deste porte, tal recomendação faz-se ainda mais importante.

No âmbito da empresa estudada, este trabalho aponta os efeitos de algumas escolhas baseadas na análise estratégica, houve falhas na primeira utilização de algumas destas ferramentas, determinando um período de desequilíbrio financeiro e que, por consequência, induziu a uma tomada de decisão crítica a respeito do futuro da empresa.

Todavia, utilizando as ferramentas que mapearam os stakeholders e competidores para determinar uma melhor percepção do ambiente de negócios, assim como o entendimento das habilidades internas por meio das ferramentas de análise estratégica, detectou-se fatores determinantes para as ações da empresa a partir daquele ponto, iniciando um processo de formulação da estratégia muito melhor embasado.

\section{REFERÊNCIAS}

AAKER, D. A. Administração Estratégica de Mercado. 9 ed. Porto Alegre: Bookman, 2009.

BARNEY, J. FIRM RESOURCES AND SUSTAINED COMPETITIVE ADVANTAGE. Journal of Management, v. 17, n. 1, p. 99-120, $\operatorname{mar} 1991$.

CANONGIA, C.; SANTOS, D. M. S.; SANTOS; M. M.; ZACKIEWICZ, M. Foresight, Inteligência Competitiva e Gestão do Conhecimento: Instrumentos para a gestão da inovação. Gestão \& Produção. v. 11, n. 2, p. 231-238, mai./ago. 2004.

CARPINETTI, L. C. R. Proposta de um modelo conceitual para o desdobramento de melhores estratégias. Gestão \& Produção. v. 7, n. 1, p. 29-42, 2000.

CAVALCANTI, C. X.; OLIVEIRA, M. P. V.; BRONZO, M. Alcançando a inovação em processo por meio da abordagem analítica. Revista de Ciências da Administração. v. 18, n. 45, p. 125-135, 2016. 
DUNNE, T. C., AARON, J. R., McDOWELL, W. C., URBAN, D. J., GEHO, P. R. The impact of leadership on small business innovativeness Journal of Business Research. 69 (2016) 4876-4881

FAHEY, L.; RANDALL, R. M. Learning from the future. New York: John Wiley \& Sons, 1998.

FERREIRA, J.J.M.; FERNANDES, C.I.; RATTEN, V. A co-citation bibliometric analysis of strategic management research. Scientometrics, v. 109, n. 1, p. 1-32, Out 2016.

FLEURY, M. T. L. et al. Competitividade e práticas de gestão em setores da indústria brasileira. 2016.

GONÇALVES, A.; KOPROWSKI, S. O. Pequena Empresa no Brasil. São Paulo: Edusp, 1995.

HARRIS, J. D; LENOX, M. J. The Strategist's Toolkit. 2013.

IMA. Measuring and managing shareholder value creation. In: SMA n. 4AA, Montvale: IMA, 1997.

LOBATO, D. M; MOYSÉS FILHO, J.; TORRES, M. C. S.; RODRIGUES, M. R. A. Estratégia de empresas. rev. Rio de Janeiro: Editora FGV, 2003.

MINTZBERG, H.; QUINN, J. B. The strategy process: concepts, contexts and cases. 2 ed. Englewood Cliffs: Prentice-Hall International, Inc., 1991.

MOLINA PALMA, M. A. A capacidade de inovação como formadora de valor: análise dos vetores de valor em empresas brasileiras de biotecnologia. Tese (Doutorado). São Paulo, FEA-USP, 2004.

ORGANIZAÇÂO PARA A COOPERAÇÃO E DESENVOLVIMENTO ECONÔMICO - OCDE. Manual de Oslo - diretrizes para a coleta e interpretação de dados sobre inovação. Brasília: FINEP, 2006.

PAIVA, M. S. DE et al. Inovação e os efeitos sobre a dinâmica de mercado: uma síntese teórica de Smith e Schumpeter. Interações (Campo Grande), v. 19, n. 1, p. 155-170, jan. 2018.

PORTER, M.E. Competitive strategy: Techniques for analyzing industry and competitors. New York: Harper \& Row, 1980

PORTER, M. E. Os caminhos da lucratividade: como implementar uma verdadeira vantagem competitiva. HSM Management. São Paulo, n. 1, p. 88-94, mar./abr. 1997.

ROCHA, W.; BORINELLI, M. L. Análise estratégica de Cadeia de Valor: um estudo exploratório do segmento indústria-varejo. Revista Contemporânea de Contabilidade. v. 1, n. 7, p. 145-165, jan./jun. 2007.

SANTOS, D. M.; SANTOS, M. M. A atividade de foresight e a União Europeia (EU). Parcerias Estratégicas. n. 17, p. 165-192, 2003.

SEBRAE - Serviço Brasileiro de Apoio às Micro e Pequenas Empresas. Panorama Sebrae. Brasília. maio, 2018.

SCHUMPETER, Joseph A. Capitalismo, Socialismo e Democracia. Rio de Janeiro: Editora Fundo de Cultura, 1961

SOUZA, C. A.; ZWICKER, R. Sistema integrado de gestão empresarial: estudos de casos de implementação de sistemas ERP. Faculdade de Economia, Administração e Contabilidade, 2000.

STEFANOVITZ, J. P.; NAGANO, M. S. Gestão da inovação de um produto: proposição de um modelo integrado. São Paulo, v. 24, n. 2, p. 462-476, abr. /jun. 2014. 
TESSARIN, M. S.; SUZIGAN, W; GUILHOTO, J. J. M. Inovação no Brasil por Intensidade Tecnológica: Cooperação e Origem do Capital. WORKING PAPER SERIES Nº 06, 2019

VOSS, C.; TSIKRIKTSIS, N; FROHLICH, M. Case research in operations management. International Journal of Operations \& Production Management. v. 22, n. 2, p. 195-219, 2002.

WOOD, D. J. Business and society. Pittsburgh: Harper Collins, 1990.

YIN, R. K. Estudo de caso: planejamento e métodos. 5 ed. Porto Alegre: Bookman, 2015. 